\title{
Application of the particle in a box electronic circuit in a spread spectrum communication system
}

\author{
José Carlos Pizolato Jr.* \\ Department of Electrical Engineering, \\ Federal University of São Carlos, \\ Rodovia Washington Luís, km 235 - SP - 310, \\ Cep: 13565-905, São Carlos, São Paulo, Brazil \\ Fax: (16) 3351-8111 \\ E-mail: jcpizolato@yahoo.com.br \\ *Corresponding author

\section{Luiz Gonçalves Neto} \\ Department of Electrical Engineering, \\ State University of São Paulo - EESC/USP, \\ Avenida Trabalhador São Carlense 400, \\ Cep: 13566-590, São Carlos, São Paulo, Brazil \\ Fax: (16) 3373-9372 \\ E-mail: lgneto@sc.usp.br
}

\begin{abstract}
A spread spectrum communication system based on the electronic model of the particle in a box system is proposed. This electronic system allows a robust and simple electronic emulation of the mechanical behaviour of the collisions of a particle inside a box, exhibiting rich chaotic behaviour. The required non-linearity to emulate the box walls is implemented in a simple way when compared with other analogue electronic chaotic circuits. In this system, a stream of binary data is transformed in the frequency modulated signal by the binary frequency shift keying (BFSK) modulator. The chaotic signal of the particle in a box electronic circuit is used to generate a broadband signal in the voltage controlled oscillator (VCO) of the transmitter. The frequency hopping signal sent to receiver is generated by mixing the binary frequency shift keying and the broadband signal. The demodulation process is done when the VCOs of transmitter and receiver are synchronised by a phase locked loop (PLL) circuit. The performance of the demodulation process is verified through the eye pattern technique applied on the recovered bit stream. A binary data stream was sent through a transmission channel with the following lengths: 2,000 metres and 8,000 metres. The experimental results demonstrated that the particle in a box electronic circuit can be used in a spread spectrum communication system.
\end{abstract}

Keywords: spread spectrum; chaotic modulation; chaotic signal.

Reference to this paper should be made as follows: Pizolato Jr., J.C. and Neto, L.G. (2011) 'Application of the particle in a box electronic circuit in a spread spectrum communication system', Int. J. Satellite Communications Policy and Management, Vol. 1, No. 1, pp.44-54.

Biographical notes: José Carlos Pizolato, Jr. received his BSc in Electrical Engineering from Escola de Engenharia de São Carlos (EESC), University of São Paulo, São Paulo, Brazil, in 1998, his MSc in Electrical Engineering 


\begin{abstract}
(chaotic modulation as an alternative to spread spectrum systems) from Escola de Engenharia de São Carlos, University of São Paulo, São Carlos, Brazil, in 2001, and his $\mathrm{PhD}$ in Electrical Engineering from EESC in 2006. He is currently a Professor in the Federal University of São Carlos (UFSCAR), São Paulo.

Luiz Gonçalves Neto received his BSc in Electrical Engineering from Escola de Engenharia de São Carlos (EESC), University of São Paulo, São Carlos, Brazil, in 1985, his MSc in Physics from Instituto de Física de São Carlos, University of São Paulo, São Carlos, in 1990, and his PhD in Physics from Centre d'Optique, Photonique et Laser, Physics Department, Laval University, Québec, QC, Canada, in 1996. He is currently an Associate Professor with the University of São Paulo, São Carlos. He is the Topical Editor of the Journal of Applied Optics.
\end{abstract}

\title{
1 Introduction
}

In conventional spread spectrum communication systems pseudorandom signals are used for broadening the spectrum by modulating the phase in direct-sequence, or the frequency in frequency hopping $(\mathrm{FH})$ of the carrier signal. This kind of modulation provides a better antijam performance, low power spectral density, and implies the reduction of multipath effects (Volkovskii et al., 2005). At the receiver a corresponding dispreading technique is used to reconstruct the baseband signal. These despreading techniques usually require synchronisation between the transmitter and the receiver.

There has been significant interest in exploiting chaotic dynamics in communications (Kennedy and Kolumbán, 2000; Oancea, 2005; Hu and Chen, 2006; Xiang-Jun, 2006; Lodi et al., 2007; Fodjouong et al., 2007; Miliou et al., 2007). Chaotic systems provide a rich mechanism for pseudo-random signal generation, with potential applications to spread spectrum communication systems (Cuomo et al., 1993; Heidari-Bateni and Gillem, 1994; Mazzini et al., 1997; Rovatti et al., 1998; Dmitriev et al., 2000; Hu and Chen, 2006). Analogue chaotic oscillators provide a natural way of generating smoothly varying frequency modulation (FM) (Volkovskii and Tsimring, 1999). In the literature there are several electronic circuits which can be used to generate chaotic signals for applications in frequency modulation. Some examples are the Chua's circuit (Alexeyev and Green, 1997; Kolumbán et al., 1998; Hu and Chen, 2006), the Lorenz-based chaotic circuit (Cuomo et al., 1993), the chaotic Rössler circuits (Garcia-Lopez et al., 2005), the particle in a box electronic circuit (Pizolato et al., 2008) and the electronic bouncing ball circuit (Zimmerman et al., 1992).

Usually chaos synchronisation is very susceptible to channel noise, distortions, and interference. To minimise the performance degradation associated with these factors, communication systems based on chaotic pulse position modulation and chaotic frequency modulation (CFM) have recently attracted much interest (Volkovskii and Tsimring, 1999; Volkovskii et al., 2005). The CFM communication scheme is a modification of the FH scheme, in which a chaotic signal is used to spread the spectrum instead of a pseudo-noise sequence. A spread spectrum communication system which uses CFM for spreading the spectrum and BFSK modulation for the information signal was proposed in reference (Volkovskii et al., 2005). In that work, the Rössler system was 
used as the chaotic oscillator. However, the analogue implementation of the chaotic circuit requires several operational amplifiers and components. Pizolato et al. (2008) presented the particle in a box electronic circuit, in that the non-linearity is implemented in a simple way and with fewer analogue devices when compared with other analogue electronic chaotic circuits.

This paper proposes the application of the particle in a box electronic circuit to implement a communication system which uses the CFM for spreading the spectrum and BFSK to modulate the information signal. In the electronic implementation of this system, the transmitter has the particle in a box electronic circuit which controls the voltage control oscillator (VCO) to generate a broadband signal. In the receiver there is a phase locked loop (PLL) circuit to perform the synchronism between the VCOs of the transmitter and receiver. The binary data stream is recovered by a frequency shift keying (FSK) demodulator that uses the resulting signal generated by the product between the VCO and the received signal. The performance of the proposed system is verified through the eye pattern technique (Keiser, 1991) applied on the binary data stream recovered at the receiver. In the demodulation process, the robustness of the spread spectrum experimental implemented was evaluated using the model $\mathrm{T}$ of the transmission line. The eye patterns of recovery binary information were measured for transmission lines with 0 metres, 2,000 metres and 8,000 metres.

This work is organised in the following sequence. In Section 2, it is discussed the electronic circuit implementation of the particle in the box electronic circuit system. This circuit will be used in the implementation of the analogue chaotic oscillator. In Section 3, the particle in the box electronic circuit is applied in an alternative spread spectrum $\mathrm{FH}$ system. In Section 4, the performance of the alternative FH system is evaluated through the eye pattern technique. Any conclusions about the experimental tests are done in Section 5. The text of the paper finishes with the references.

Figure 1 The particle in a box electronic circuit described by equation (1)

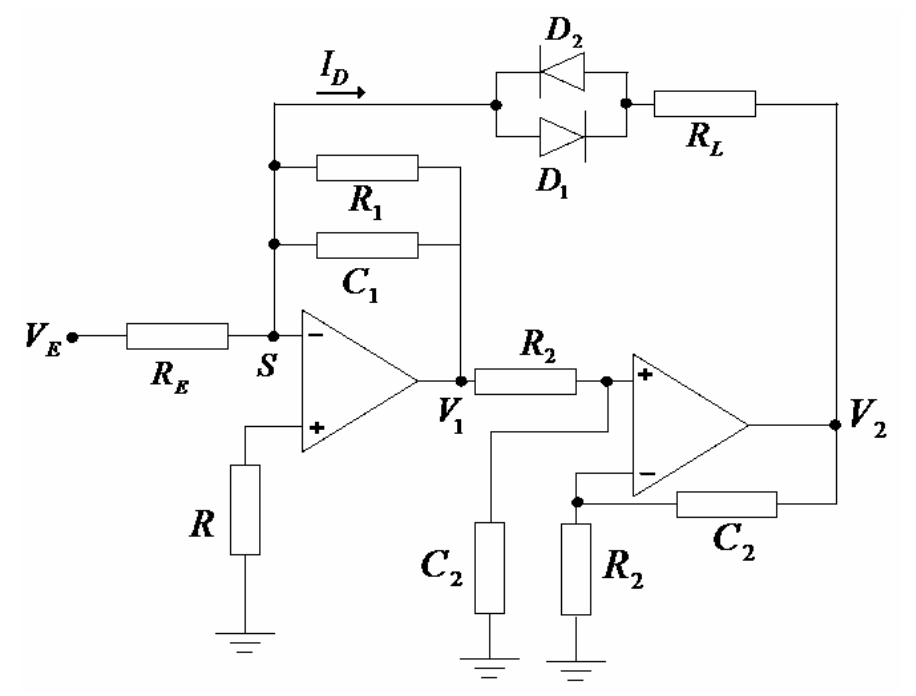

Notes: The component values are: resistors $(k \Omega): \mathrm{R}_{1}=47 ; \mathrm{R}=\mathrm{R}_{\mathrm{E}}=\mathrm{R}_{2}=10 ; \mathrm{R}_{\mathrm{L}}=0.51$; capacitors $(\mathrm{nF}): \mathrm{C}_{1}=\mathrm{C}_{2}=12$; diodes $\mathrm{D}_{1}$ e $\mathrm{D}_{2}$ : 2N4148; operational amplifiers: 741 or equivalents. 


\section{The analogue chaotic oscillator}

The particle in a box electronic circuit (Pizolato et al., 2008) described in Figure 1 was implemented with electronic components. This circuit simulates the mechanical behaviour of the collisions of a particle inside a box. It is based on the 'electronic bouncing ball circuit', proposed by (Zimmerman et al., 1992), which is used to study the regular, chaotic and random behaviour of a bouncing ball on a vibrating surface. The key devices of the particle in a box electronic circuit are the anti-parallel diodes $D_{1}$ and $D_{2}$ and the resistor $R_{L}$. They electronic implement the non-linearity this circuit in a simple way when compared with other circuits (Cuomo et al., 1993; Kolumbán et al., 1998; Alexeyev and Green, 1997). The association of these devices simulates the collision of the particle in walls in the $x$ direction, resulting in the term $I_{D}\left(V_{2}\right)$ plotted in Figure 2.

Figure 2 Curve of current $I_{D}\left(V_{2}\right)$ versus voltage $V_{2}$ of the anti-parallel diodes $\left(D_{1}\right.$ and $\left.D_{2}\right)$ in series with the resistor $R_{L}$ of the particle in a box electronic circuit

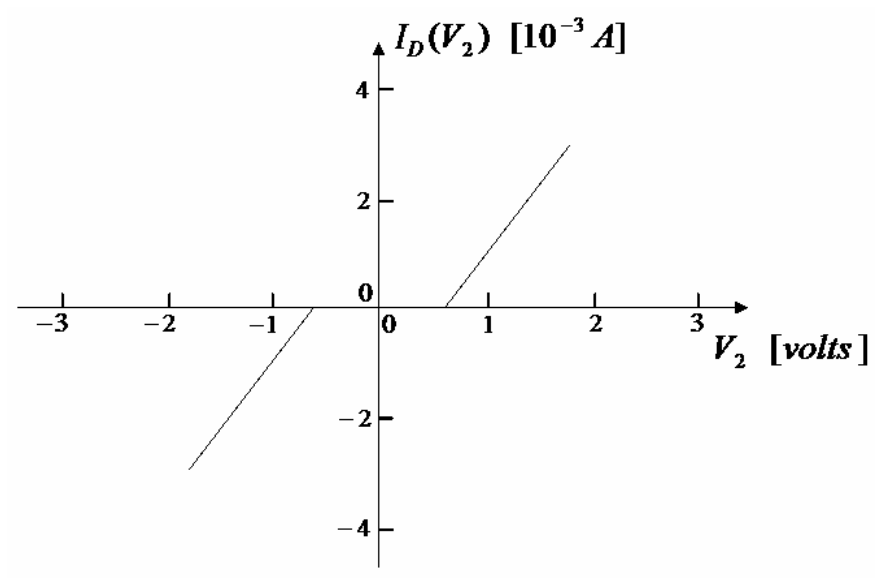

The particle in a box electronic circuit has as input a sinusoidal signal $V_{E}=V_{\max } \sin (w t)$, and the contribution of the currents in the point $S$ of Figure 2, results in the differential equation:

$$
R_{2} C_{1} C_{2} \frac{d^{2} V_{2}}{d t^{2}}+\frac{R_{2} C_{2}}{R_{1}} \frac{d V_{2}}{d t}+I_{D}\left(V_{2}\right)=-\frac{V_{E}}{R_{E}}
$$

In the equation (1), the signal $V_{2}$ corresponds to the position $x$ of the system described in Figure 1 , and the signal $d V_{2} / d t$ corresponds to the velocity $d x / d t$. The component values are resistor $(\mathrm{k} \Omega): \quad R_{1}=47 ; R=R_{E}=R_{2}=10 ; \quad R_{L}=0.51 ; \quad$ capacitors $(\mathrm{nF})$ : $C_{1}=C_{2}=12$; Diodes $\mathrm{D}_{1}$ and $\mathrm{D}_{2}$ : 2N4148; operational amplifiers: CI 741 or equivalents. The components of the operational amplifiers can be seen in the reference (Graeme et al., 1981). All resistors and capacitors used in this work have an error of $+/-10 \%$ in their nominal values.

The periodic or chaotic behaviour can be obtained by the variation of the bifurcation parameter of the circuit of Figure 1, which controls the dynamic of the system. The variation of the parameters $w$ or $V_{\max }$ of the input sinusoidal signal $V_{E}=V_{\max } \sin (w t)$ can be used as the bifurcation parameters. As a first example to show the dynamic of the 
chaotic behaviour of the particle in a box system, the amplitude $V_{\max }$ has been chosen as bifurcation parameter. The initial conditions of this circuit were $\left(V_{2}=0\right.$ and $\left.d V_{2} / d t=0\right)$. The variation of $V_{\max }$ can causes a periodic oscillation with period $T$, passing by a period doubling bifurcation, respectively with period $2 T$ and $4 T$, until a non-periodic chaotic oscillation. In the electronic simulation of this system, the period doubling bifurcation is limited to $4 T$ due to the noisy level present in the circuit (Zimmerman et al., 1992). This period doubling behaviour followed by the chaotic oscillations is shown in the phase diagrams of Figure 3.

Figure 3 Phase diagrams of the particle in a box electronic circuit for the fixed oscillating frequency $w=13,000 \mathrm{rad} / \mathrm{s}$ with the initial conditions $\left(V_{2}=0\right.$ and $\left.d V_{2} / d t=0\right)$ :

(a) period 1 (b) period 2 (c) period 4 (d) chaos

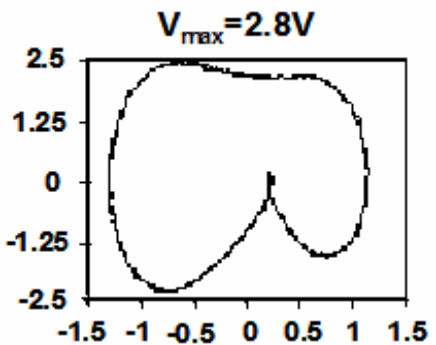

(a)

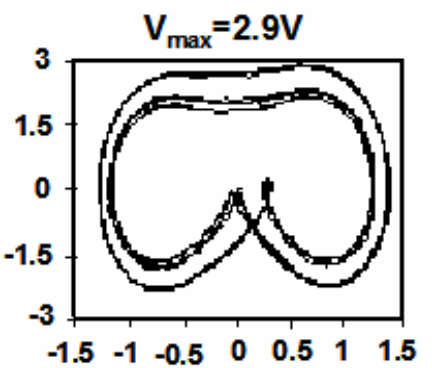

(c)

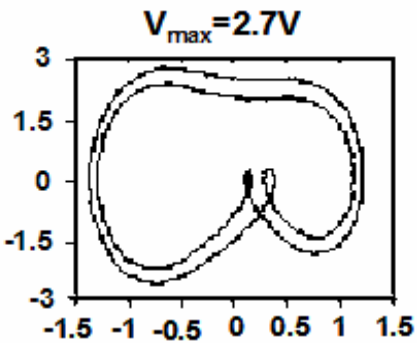

(b)

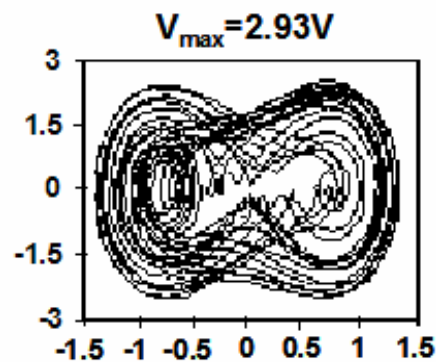

(d)

The particle in the box electronic circuit has been used in an experimental implementation of a communication system (Pizolato, Romero and Neto, 2008). This circuit is implemented in a simple way and with fewer analogue devices when compared with other analogue electronic chaotic circuits. In this work it is proposed the application these circuit as the analogue chaotic oscillator in an alternative spread spectrum $\mathrm{FH}$ system.

\section{The particle in the box electronic circuit in a spread spectrum system}

A conventional spread spectrum system by $\mathrm{FH}$ is illustrated in Figure 4. A frequency generator generates a carrier with a random frequency using a pseudo random sequence. 
The recovery process of the information is obtained when the receiver synchronises with the transmitter. The carrier signal has a frequency in each instant and it has a wideband in frequency domain.

Figure 4 Scheme of a spread spectrum system by FH

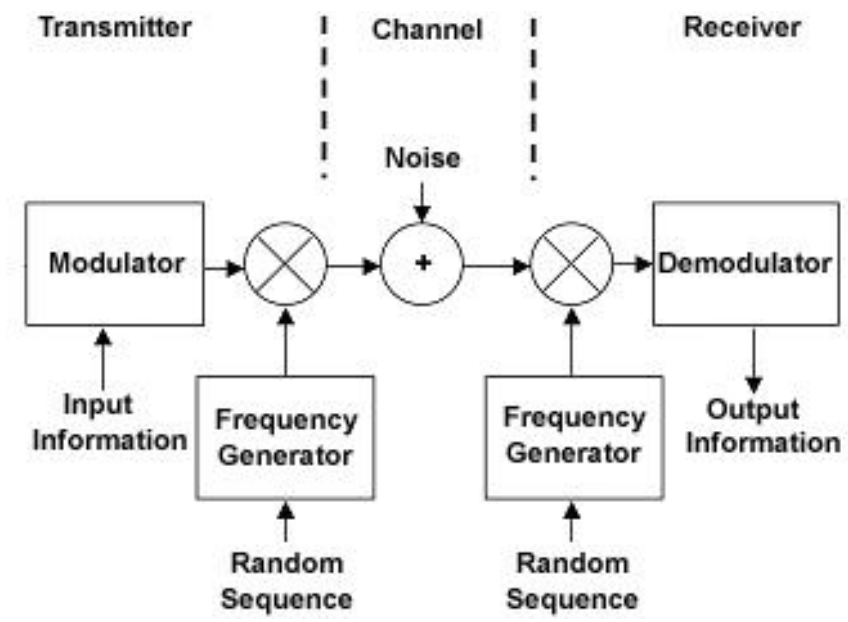

An alternative way to generate the carrier with random frequency applies an analogue chaotic oscillator. The block-diagram of this system is shown in Figure 5. The spreading of information in the transmitter is done by the particle in the box electronic circuit of Figure 1. The input sinusoidal signal $V_{E}=V_{\max } \sin (w t)$ is chosen to get a phase diagram of Figure 3(d) with $V_{\max }=2.93 \mathrm{~V}$ and $w=13,000 \mathrm{rad} / \mathrm{s}$ to the initial conditions $\left(V_{2}=0\right.$ and $\left.d V_{2} / d t=0\right)$. The chaotic signal $V_{1}(t)$ in the output of the second operational amplifier of this circuit is illustrated in Figure 6. In this case $V_{1}(t)$ is a wideband signal with a chaotic behaviour and it is applied in the input of the voltage controlled oscillator of the transmitter $\left(\mathrm{VCO}_{\mathrm{t}}\right)$ to generates a sinusoidal carrier $V_{F H r}(t)$ with chaotic variable frequency. The VCOs blocks were electronic implemented with CI ICL8038. The frequency of $\mathrm{VCO}_{\mathrm{t}}$, modulated by the chaotic signal $V_{1}(t)$, is described by equation (2).

$$
\dot{\varphi}_{t} \equiv w_{t}=w_{o}\left(1+m_{1} V_{1}\right)
$$

in which $w_{o}$ is the 'natural' frequency of $\mathrm{VCO}_{\mathrm{t}}, \mathrm{m}_{1}$ is the modulation gain coefficient $\left(\mathrm{m}_{1}=0.11\right)$ and $V_{1}(t)$ is the chaotic signal.

The input information $d(t)$ (a binary data stream ) is applied in the FSK modulator generating the BFSK signal $S_{t}(t)$ of Figure 7(a) with the power spectrum as shown in Figure 7(b). The FSK modulator block was electronic implemented with CI ICL8038. The BFSK signal and the chaotic sinusoidal carrier are multiplied by the phase discriminator block $(\otimes)$ resulting in the spread spectrum signal of Figure 8 . The block $(\otimes)$ was electronic implemented with CI MM74C932. The signal $V(t)$ of Figure 8 has a wideband from $20 \mathrm{kHz}$ to $60 \mathrm{kHz}$ and it is described by equation (3).

$$
V(t)=P_{s} \cos \left(w_{i} t+\Omega t d(t)+\theta\right)
$$

in which $P_{S}$ is the amplitude of the signal $S_{t}, w_{i}$ is carrier frequency, $d(t)$ is the binary data stream information, $\Omega$ is a parameter of the FSK modulator and $\theta$ is a phase factor. 
Figure 5 Block diagram of the alternative spread spectrum using an analogue chaotic oscillator (the particle in the box electronic circuit)

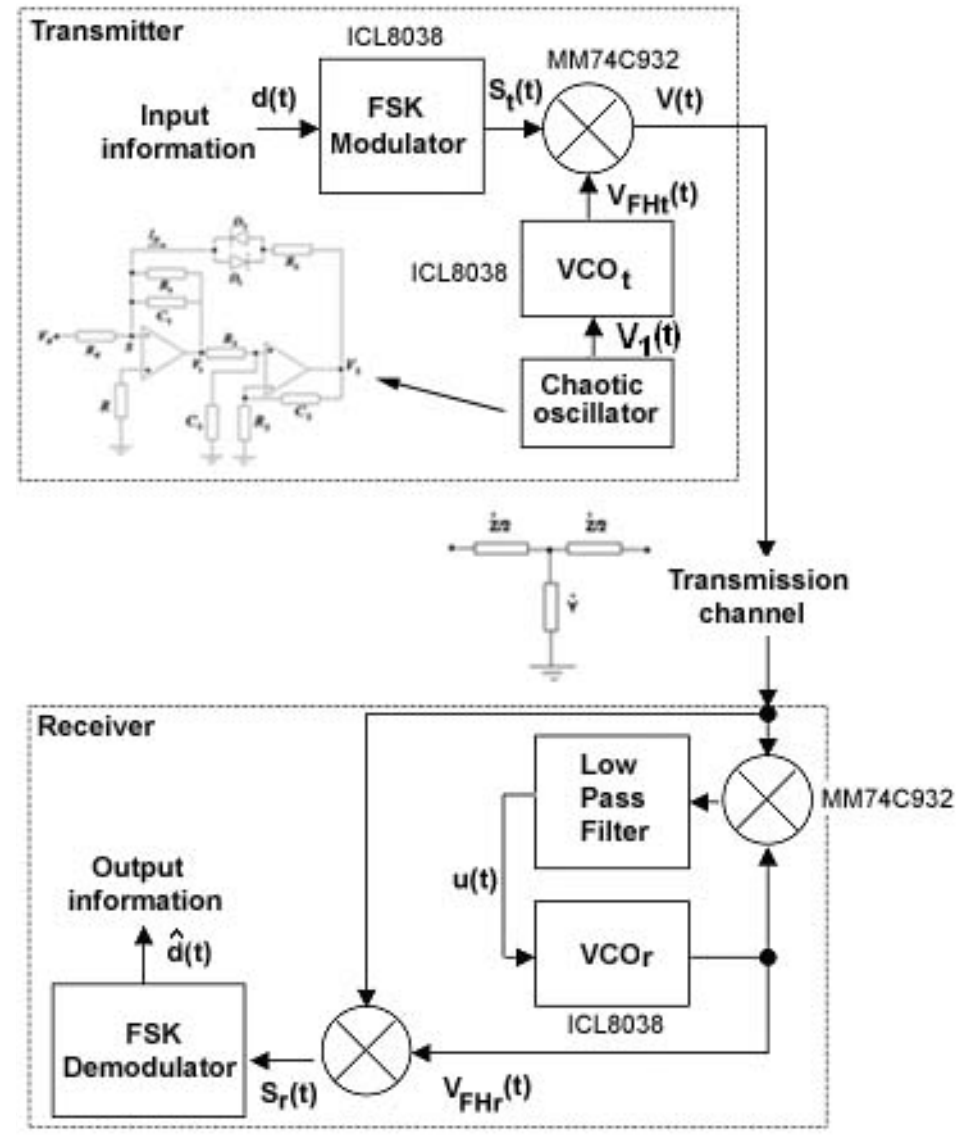

Figure 6 Chaotic signal $V_{1}$ of the particle in the box electronic circuit with the initial conditions $\left(V_{2}=0\right.$ and $\left.d V_{2} / d t=0\right)$

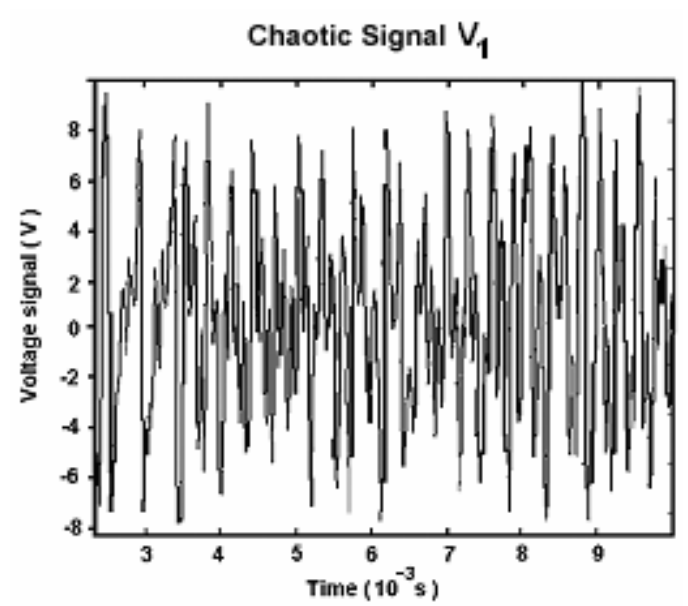


Figure 7 BFSK modulation (a) BFSK signal (b) power spectrum of the BFSK signal

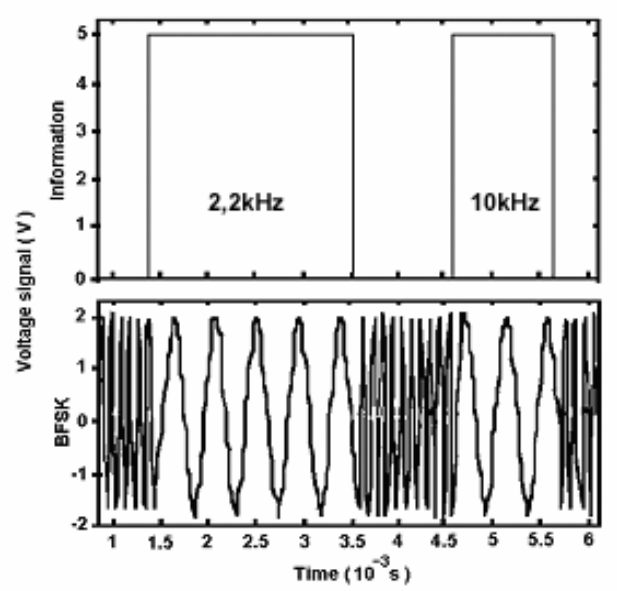

(a)

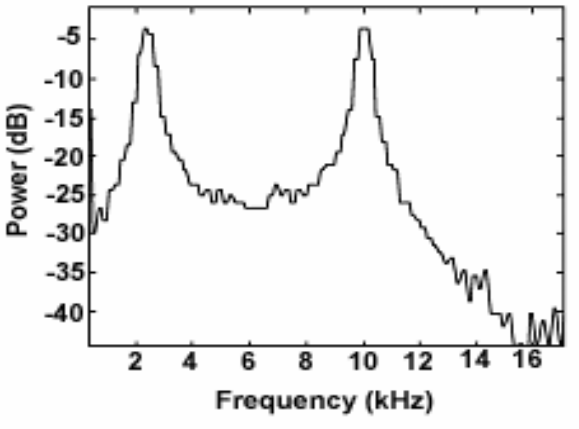

(b)

Figure 8 Power spectrum of the spread spectrum signal $V_{1}(t)$

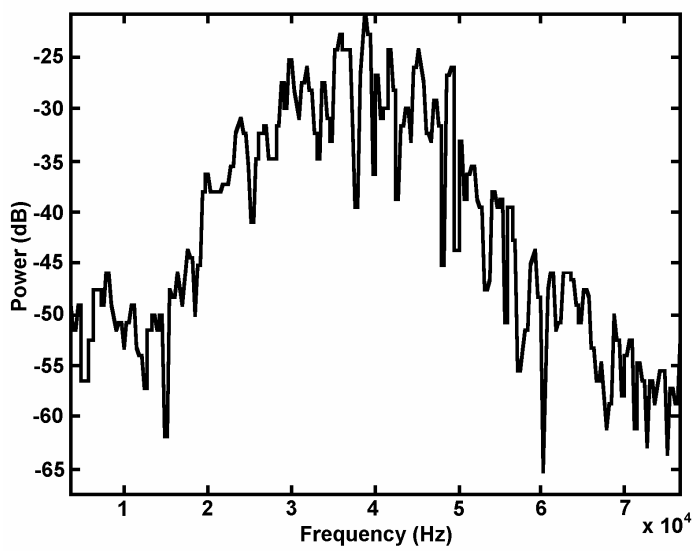

The local $\mathrm{VCO}_{\mathrm{r}}$, the phase discriminator $(\otimes)$ and the low pass filter (LPF) form a PLL. The LPF has the transfer function done by equation (4).

$$
K(s)=1 /\left(1+T_{f} s\right)
$$

in which $T_{f}$ is the time constant of the filter.

The frequency detection is performed using the PLL that synchronises transmitter and receiver. In this case the frequency of $\mathrm{VCO}_{\mathrm{r}}$ is described by equation (5).

$$
\dot{\varphi}_{r} \equiv w_{r}=w_{o}\left(1+m_{2} u(t)\right)
$$

in which $\mathrm{m}_{2}$ is the modulation gain coefficient $\left(\mathrm{m}_{2}=0.12\right)$ and $u(t)$ is the output signal of the LPF.

If the PLL is able to compensate the frequency deviations and keep the VCOs synchronised, the sinusoidal carrier with chaotic variable frequency $w_{r} \cong w_{t}$ can be 
recovered in the receiver. After, the BFSK signal $S_{r}(t)$ is recovered using the phase discriminator block $(\otimes)$ and a FSK demodulator is used to recovery the binary data stream $d(t)$ from $S_{r}(t)$ signal. The block FSK demodulator was electronic implemented with CI LM565.

\section{An alternative spread spectrum FH}

A spread spectrum system of Figure 5 was electronic implemented as described in Section 3. A random binary data stream of 512 bits is used like input information signal $d(t)$. The transmission channel was implemented by a transmission line model $\mathrm{T}$ with 0 metres, 2,000 metres and 8,000 metres of length. The line model $\mathrm{T}$ was implemented with resistors, inductors and capacitors. At the receiver the input information signal $\hat{d}(t)$ is recovered of spread spectrum signal $V(t)$.

Figure 9 Eye pattern of the binary data stream recovered (a) transmission line of 0 metres (b) transmission line of 2,000 metres $\left(Z_{1}=6,5+j 2,6 \Omega\right.$ and $Y_{1}=j 0,00103$ Siemens $)$ (c) transmission line of 8,000 metres $\left(Z_{2}=26+j 10,4 \Omega\right.$ and $Y_{1}=j 0,00826$ Siemens $)$

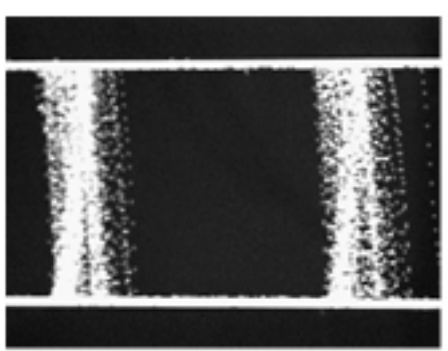

Transmission line ( 0 meters )

(a)

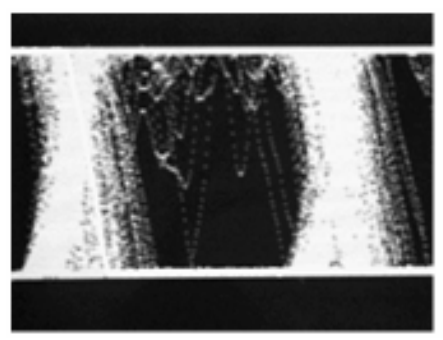

Transmission line ( 2000 meters )

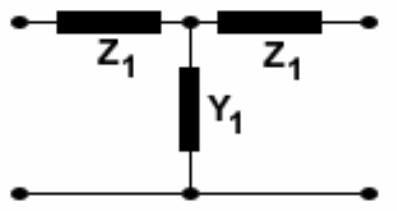

(b)

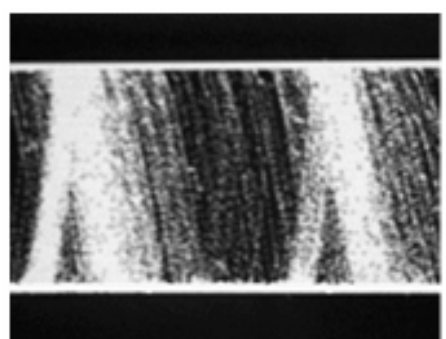

Transmission line ( 8000 meters )

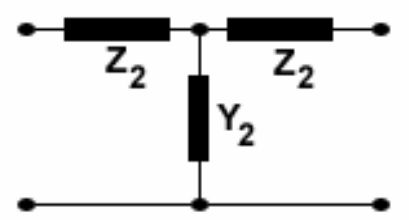

(c) 
The eye pattern technique (Keiser, 1991) is used to verify the performance of the recovery process of the binary data stream. A recovery process without bit error should have a pattern like an opened eye. Figure 9(a) illustrates the recovery process with transmission line like a short line circuit. The pattern is an opened eye because the communication process does not present high level of bit error. Figure 9(b) illustrates other recovery process with transmission line of 2,000 metres. In this figure the eye is closer than the last case because of the bit errors introduced by the length of the transmission line. Figure 9(c) illustrates the recovery process with transmission line of 8,000 metres. In this case, the eye pattern is closer than the case with transmission line of 2,000 metres. The recovery process presents problems such as high amplitude distortion, temporisation errors and phase distortion (timing jitter). These eyes pattern show the influence of the length of transmission line in the quality of detection.

\section{Conclusions}

A possible approach to spread spectrum communication system was demonstrated. In this paper it was implemented a spread spectrum communication system FH that uses an analogue chaotic circuit. The particle in a box electronic circuit was used like the analogue chaotic circuit. The rich chaotic behaviour of this circuit was used in to generate a sinusoidal carrier with random variable frequency. This circuit was chosen because it is implemented in a simple way and with fewer analogue devices when compared with other analogue electronic chaotic circuits. The performance of the systems was verified through the eye pattern of the recovered binary data stream. The recovery process presented problems such as high amplitude distortion, temporisation errors and phase distortion (timing jitter). The eyes pattern showed the influence of the length of transmission line in the quality of detection. The experimental results in this paper show that the particle in a box electronic circuit is an interesting alternative to implement a spread spectrum communication system.

\section{Acknowledgements}

The authors would like to acknowledge the many helpful suggestions of anonymous reviewers. We also thank the State University of São Paulo and State University of Londrina for the financial support.

\section{References}

Alexeyev, A.A. and Green, M.M. (1997) 'Secure communications based on variable topology of chaotic circuits', International Journal of Bifurcation and Chaos, Vol. 7, No. 12, pp.2861-2869.

Cuomo, K.M., Oppenheim, A.V. and Strogats, S.H. (1993) 'Synchronization of Lorenz-based chaotic circuits with applications to communications', IEEE Transactions on Circuits and Systems II : Analogue and Digital Signal Processing, Vol. 40, No. 10, pp.626-633.

Dmitriev, A.S., Panas, A.I. and Starkov, S.O. (2000) 'Multiple access communication based on control of special chaotic trajectories', Institute of Radio Engineering and Electronics Russian Academy of Sciences Mochovaya st. 11, GSP-3, Moscow, Russia. 
Fodjouong, G.J., Fotsin, H.B. and Woafo, P. (2007) 'Synchronization modified van der Pol-Duffing oscillators with offset terms using observer design: application to secure communications', Physica Scripta, Vol. 75, No. 5, pp.638-644.

Garcia-Lopez, J.H., Reátegui, R.J., Pisarchik, A.N., Hernandez, A.M., Gutiérrez, C.M., Hernandez, R.V. and Rauda, V. (2005) 'Novel communication scheme based on chaotic Rössler circuits', International Conference on Control and Synchronization of Dynamical Systems, Journal of Physics: Conference Series, Vol. 23, pp.276-284.

Graeme, J.G., Tobey, G.E. and Huelsman, L.P. (1981) Operational Amplifiers Design and Applications, McGraw-Hill, International Student edition.

Heidari-Bateni, G. and Mc Gillem, C.D. (1994) 'A chaotic direct sequence spread spectrum communication system', IEEE Transactions on Communications, Vol. 42, Nos. 2/3/4, pp.1524-1527.

$\mathrm{Hu}, \mathrm{Z}$. and Chen, X. (2006) 'Synchronization of chaotic cryptosystems based on Chua's circuits with key functions', Dynamics of Continuous Discrete and Impulsive Systems - Series A Mathematical Analysis, Vol. 13, pp.489-499.

Keiser, G. (1991) Optical Fiber Communications, McGraw-Hill, 2nd ed.

Kennedy, M.P. and Kolumbán, G. (2000) 'Special issue on non-coherent chaotic communications', IEEE Transactions on Circuits and Systems-I: Fundamental Theory and Applications, Vol. 47, No. 12, pp.1661-1662.

Kolumbán, G., Kennedy, M.P. and Chua, L.O. (1998) 'The role of synchronization in digital communications using chaos - part II: chaotic modulation and chaotic synchronization', IEEE Transactions on Circuits and Systems-I: Fundamental Theory and Applications, Vol. 45, No. 11, pp.1129-1140.

Lodi, V.A., Benedetti, M., Merle, S., Perez, T., Colet, P. and Mirasso, R. (2007) 'Message encryption by phase modulation of a chaotic optical carrier', IEEE Photonics Techonology Letters, Vol. 19, No. 2, pp.76-78.

Mazzini, R., Setti, G. and Rovatti, R. (1997) 'Chaotic complex spreading sequences for asynchronous DS-CDMA - part I: system modeling and results', IEEE Transactions on Circuits and Systems-I: Fundamental Theory and Applications, Vol. 44, No. 10, pp.937-947.

Miliou, A.M., Antoniades, I.P., Stavrinides, S.G. and Anagnostopoulos, A.N. (2007) 'Secure communication by chaotic synchronization: robustness under noisy conditions', Nonlinear Analysis : Real World Applications, Vol. 8, No. 3, pp.1003-1012.

Oancea, S. (2005) 'Synchronization of chaotic electronic Sprotts's circuits', Journal of Optoelectronics and Advanced Materials, Vol. 7, No. 6, pp.2919-2923.

Pizolato Jr., J.C., Romero, M.A. and Neto, L.G. (2008) 'Chaotic communication based on the particle-in-a-box electronic circuit', IEEE Transactions on Circuits and Systems-I: Regular Papers, Vol. 55, No. 4, pp.1108-1115.

Rovatti, R., Setti, G. and Mazzini, G. (1998) 'Chaotic complex spreading sequences for asynchronous DS-CDMA - part II: some theoretical performance bounds', IEEE Transactions on Circuits and Systems-I: Fundamental Theory and Applications, Vol. 45, No. 4, pp.496-506.

Volkovskii, A.R. and Tsimring, L.S. (1999) 'Synchronization and communication using chaotic frequency modulation', International Journal of Circuit Theory And Applications, Vol. 27, No. 6, pp.569-576.

Volkovskii, A.R., Tsimring, L.Sh., Rulkov, N.F. and Langmore (2005) 'Spread spectrum communication system with chaotic frequency modulation', Chaos, Vol. 15, pp.033101-1-033101-6.

Xiang-Jun, W. (2006) 'A new chaotic communication scheme based and adaptive synchronization', Chaos, Vol. 16, No. 4, pp.043118-1-043118-12.

Zimmerman, R.L., Celaschi, S. and Neto, L.G. (1992) 'The electronic bouncing ball', American Journal of Physics, Vol. 60, No. 4, pp.370-375. 